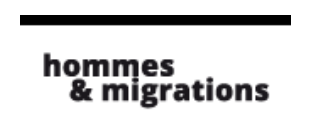

\section{Hommes \& migrations}

Revue française de référence sur les dynamiques

migratoires

$1296 \mid 2012$

Le Mexique dans les migrations internationales

\title{
Frontières territoriales et frontières symboliques
}

L'immigration au prisme de l'action publique française et italienne

\section{Annalisa Lendaro}

\section{(2) OpenEdition \\ 1 Journals}

\section{Édition électronique}

URL : http://journals.openedition.org/hommesmigrations/1521

DOI : 10.4000/hommesmigrations. 1521

ISSN : 2262-3353

Éditeur

Musée national de l'histoire de l'immigration

Édition imprimée

Date de publication : 1 mars 2012

Pagination : 118-125

ISSN : 1142-852X

\section{Référence électronique}

Annalisa Lendaro, «Frontières territoriales et frontières symboliques », Hommes \& migrations [En ligne], 1296 | 2012, mis en ligne le 29 mai 2013, consulté le 22 septembre 2020. URL : http:// journals.openedition.org/hommesmigrations/1521 ; DOI : https://doi.org/10.4000/ hommesmigrations. 1521

Ce document a été généré automatiquement le 22 septembre 2020.

Tous droits réservés 


\title{
Frontières territoriales et frontières symboliques
}

L'immigration au prisme de l'action publique française et italienne

\author{
Annalisa Lendaro
}

1 La mise en œuvre des politiques publiques nécessite le classement de leurs populations cibles. Or les catégories créées par l'action publique ne sont pas neutres. Dans le cas de deux régions française et italienne, la Paca et la Ligurie, l'utilisation de la catégorie d'“immigrés" dépend des représentations que les acteurs publics se forment de certains ressortissants étrangers. La distinction entre immigrés débouche sur des politiques, comme la lutte contre les discriminations, qui demeurent à géométrie variable.

2 Cette thèse en sociologie ${ }^{1}$ questionne l'action publique ${ }^{2}$ dans sa multiple et ambivalente faculté à catégoriser l'immigré(e) et à légitimer son intervention sur les enjeux qui lui sont associés, à travers l'analyse critique des catégories produites dans le domaine de l'emploi. L'accent mis sur ce domaine se justifie doublement par l'acuité des enjeux liés à la régulation du marché du travail, notamment dans une période de chômage de masse, puis par les recompositions multiples d'une action publique qui se traduisent par la multiplication des espaces de gouvernance, y compris territoriaux. La généalogie et l'évolution sociétale des catégories de l'immigration sont appréhendées avec une double entrée : d'une part, le territoire, dont l'échelle comparative privilégiée (mais pas unique) est la région ${ }^{3}$; le deuxième registre est sectoriel, car l'analyse se développe à partir du cas de deux secteurs professionnels traditionnellement ouverts à cette maind'œuvre, que sont le bâtiment et l'aide à domicile.

3 Frontières territoriales et frontières symboliques s'entrecroisent $t^{4}$, souvent de manière conflictuelle et paradoxale, et concourent à la construction de catégories de l'immigration à différents niveaux : institutionnel d'abord, car les politiques publiques disposent d'un pouvoir de définition à valeur juridique et normative; puis organisationnel lorsqu'il s'agit d'enjeux plus spécifiquement sectoriels, au niveau de la branche ou de l'entreprise; et, parallèlement, au niveau des constructions sociales et des pratiques des individus qui s'accommodent, interprètent, transforment, ignorent les catégories construites par l'action publique et qui mettent en tension les parcours 
types qu'elle dessine. En créant des parcours types, l'action publique démontre sa capacité à construire des catégories sociales morales, définissant un ensemble de droits et d'obligations, des règles de conduite, donc des modèles d'insertion considérés comme préférables à d'autres.

Notre analyse prend en compte, d'une part, le rôle des institutions dans leur pouvoir de construction des catégories officielles de l'immigration, notamment dans la sphère de la régulation du marché du travail ; d'autre part, les processus de réinterprétation des acteurs en charge de la mise en œuvre des politiques et de certains acteurs du marché du travail (comme les intermédiaires de l'emploi), dans le cadre de négociations interpersonnelles. Ensuite, c'est dans les transitions et les bifurcations des parcours biographiques, s'apparentant à des formes de bricolages plus qu'aux trajectoires types dessinées par les dispositifs publics, que nous avons cherché, comme dans un jeu de miroirs, le rôle pluriel et ambivalent de l'action publique.

La reconstruction des parcours des travailleur(se)s immigré(e)s, qui fait l'objet du dernier chapitre de cette thèse, montre qu'une personne, selon les contextes au sein desquels elle se déplace, peut traverser une multitude de statuts différents, plus ou moins formalisés par des catégories légales, en passant de l'“immigré régulier" à l'“irrégulier", du "clandestin" au "régularisé", de l'“étranger" au "naturalisé", de l' extracomunitario à l'“"Européen", etc. Le rôle des politiques publiques est déterminant dans la mesure où elles créent des catégories-cible légitimes ouvrant l'accès à des statuts porteurs de droits ${ }^{5}$, et pas seulement de représentations.

6 Ainsi, plus que la figure de l'étranger, celle(s) de l“'immigré(e)" avec ou sans papiers, traverse les pages de cette thèse et fait émerger les contradictions d'une action publique de plus en plus territorialisée ${ }^{6}$ mais aux prises avec la mondialisation de certains enjeux, comme la circulation de la main-d'œuvre?

\section{Pour une sociologie des catégories de l'immigration : comment peut-on être immigré(e)?}

7 Les phénomènes migratoires prennent forme et évolent au sein de contextes à la fois transnationaux ${ }^{8}$ et locaux ${ }^{9}$, où l'action publique et ses protagonistes (les décideurs mais aussi les acteurs de la mise en œuvre, de la société civile, etc.) jouent un rôle déterminant dans la définition de qui est un(e) immigré(e) et de ce qui ne l'est pas. Cette action publique est créatrice de frontières : il s'agit moins de l'invention de nouvelles formes de séparation entre territoires que de la construction de catégories qui définissent les profils de populations qui peuvent devenir la cible des politiques. Nous avons pu montrer, notamment grâce à la comparaison, que ces catégories n'ont rien de "naturel" ou de "donné", même (voire surtout) lorsqu'elles sont officielles. Alors que les catégories inscrites dans les lois (nationales et supranationales) et dans les politiques publiques sont souvent envisagées de manière statique et normative, dans le sens d'une influence presque déterministe de l'intervention publique sur la mise en œuvre de régulations plus territoriales et sur les comportements individuels, nous avons souhaité faire ressortir les marges de manœuvre des acteurs de la mise en œuvre et des individus en interaction, du point de vue de leur capacité (et souvent de leur besoin) à réinterpréter ou à contourner les catégories officielles, pour en construire 
d'autres ou pour leur donner un sens et une légitimité adaptés à des circonstances particulières.

8 Le concept de frontière et celui de catégorie devraient à notre avis faire l'objet d'une ample réflexion pluridisciplinaire : une fois traversée une frontière externe, d'autres frontières plus ou moins visibles se manifestent, transformant, multipliant, dissociant, et parfois même recomposant l'identité juridique et sociale du migrant lui-même ${ }^{10}$; son inclusion dans des catégories officielles se révèle en ce sens déterminante pour le déroulement de son parcours d'insertion socioprofessionnelle.

9 Nous avons analysé l'action publique en partant du principe selon lequel il y aurait moins des "problèmes posés par les personnes immigrées" que des "questions relatives à l'immigration". En ce sens, l'immigration concerne autant les Français et les Italiens de souche, que les immigré(e)s. Si des catégories officielles existent, donnant lieu à des nomenclatures et des codages de la réalité relativement stables ${ }^{11}$, la catégorisation est également un processus plus commun et intuitif, une opération quotidienne de l'individu qui vise à réduire la complexité du monde par le biais de distinctions et de séparations. Le va-et-vient entre les échelles de l'action publique montre que les catégories officielles de l'immigration sont parfois en décalage, voire incompatibles et contradictoires, avec les catégories de la mise en œuvre mais surtout avec les catégories indigènes de l'interaction individuelle. Un des apports de notre thèse est d'avoir montré que leur utilité et leur légitimité sont constamment redéfinies au sein de contextes organisationnels et configurationnels spécifiques.

10 Ce qui rend instables et contradictoires les catégories de l'immigration est le fait qu'il peut y avoir des "immigrés sans immigration" (par exemple, lorsqu'on parle de "fils d'immigrés"), et de l"immigration sans immigrés" (dans le cas des ressortissants canadiens ou japonais, par exemple). Ainsi, les catégories officielles ne suffisent pas pour définir ce qu'est un immigré ou un extracomunitario. Ces termes font en effet l'objet d'usages sociaux plus étendus, dans des contextes multiples.

11 En France comme en Italie, des populations qui théoriquement rentrent parfaitement dans la définition officielle d"'immigré" ne sont jamais associées à cette catégorie. Le terme d'“immigré" est alors utilisé pour désigner surtout les étrangers (ou éventuellement les Français par naturalisation) originaires de certains pays, notamment des ex-colonies ou des pays perçus comme étant moins développés économiquement. Sont alors immigrés les Marocains, les Antillais et les "jeunes de banlieues ${ }^{12 "}$ ", mais pas les Australiens.

12 En Italie, le succès de la catégorie d'extracomunitario n'est pas moins paradoxal dans la mesure où on aura tendance à y inclure des individus de nationalité roumaine, même si la Roumanie fait partie depuis 2007 de l'Union européenne, mais pas les Canadiens.

13 L'immigré est ainsi le produit d'une construction sociale. Ces catégories montrent qu'il y aurait des "immigrés" plus "immigrés" que d'autres; toutefois, pour décider si un individu est un immigré ou pas, on a besoin de le comparer à des prototypes de la catégorie, censés fournir des repères cognitifs à la personne ou à l'institution "catégorisatrice". 


\section{Le pouvoir heuristique de la comparaison}

Nous nous sommes proposés de commencer par "chausser les lunettes surplombantes" des politiques publiques européennes et nationales, avec leurs catégories juridiques et officielles, pour ensuite chausser les "lunettes du local", où la focale se restreint et permet de voir de plus près une multitude d'acteurs à compétences différentes, interagissant selon des modalités de coordination typiquement sociétales ${ }^{13}$, produisant alors des variantes locales des catégories formelles. Plusieurs niveaux de comparaison se sont imbriqués, pour montrer que les catégories qui émergent de négociations et compromis entre acteurs relèvent de cohérences sociétales inscrites dans le temps, et font émerger des problèmes sociaux et des instruments d'action légitimes sociétalement.

15 Notre étude nous permet également d'avancer que le "sociétal" ne se boucle pas nécessairement au niveau national ${ }^{14}$. Pour chaque région (la région Provence-AlpesCôte-d'Azur pour la France et la région Ligurie pour l'Italie), le caractère sectoriel de l'action publique a également été considéré : nous avons étudié en profondeur les catégories construites dans les espaces de négociation de branche telles que le bâtiment et les services à la personne.

La portée heuristique d'une comparaison internationale est apparue nettement: le territoire régional s'est révélé une échelle clef d'un espace multiniveaux de déclinaison et de construction de catégories de "travailleur(se) immigré(e)". L'apport théorique de cette thèse concerne notamment l'intérêt d'articuler l'approche de l'analyse sociétale "revisitée" (attentive à la socialisation des éléments de la comparaison) avec celle de la théorie des conventions (utile pour comprendre les logiques de justification des acteurs inscrits dans un contexte précis). En adoptant une double focale "organisationnelle" (attentive aux modes de fonctionnement interne à un organisme, par exemple Pôle emploi) et "configurationnelle" ("qui travaille avec qui", et selon quelles modalités), nous avons pu faire émerger le caractère éminemment conventionnel des catégories de l'immigration, en montrant qu'elles résultent de négociations, de jeux de pouvoir, et de compromis qui les rendent plus ou moins légitimes.

\section{Les "statistiques ethniques" et les dispositifs de lutte contre les discriminations : des catégories d'action sociétalement situées}

17 Le cas de la région Paca et celui de la région Ligurie témoignent d'une décentralisation évidente de l'action publique, par ailleurs complétée par une régulation supranationale (européenne notamment) qui intervient dans les deux contextes à la fois en tant qu'instrument financier et comme "label" légitimant de nombreuses initiatives locales dans le domaine de la lutte contre les discriminations, de la promotion de l'“intégration" et de l'accès aux droits sociaux des immigrés.

18 À partir de ce point commun, on pourrait s'attendre à ce que dans les deux régions apparaissent des processus similaires de fabrication locale d'instruments statistiques qui justifient de la mise en place de ces initiatives et qui permettent d'en évaluer l'impact sur le territoire. Or, il n'en est rien. 
19 En région Paca, le processus de décentralisation s'accompagne d'une déconcentration importante des services de l'État, très concernés par la mise en œuvre territoriale des politiques qui visent à réguler l'immigration et à lutter contre les discriminations. Cela explique en partie le fait que l'élaboration à une échelle régionale d'outils statistiques alternatifs à ceux des organismes publics nationaux est difficile. Le sujet de l'immigration ne se prête pas à servir de terrain d'entente pour d'éventuelles expérimentations locales, car la méfiance vis-à-vis de catégories renvoyant à l'ethnicité n'aide pas à l'émergence d'un consensus élargi sur un renouveau des outils statistiques.

Pour qu'un codage territorial du réel devienne opérationnel il est fondamental que les négociations entre acteurs concernés (experts, décideurs, administrations déconcentrées, collectivités territoriales, associations, etc.) aboutissent à des compromis sur ce qui est légitime de mesurer et avec quels instruments ${ }^{15}$. Mais, en Paca, le référentiel républicain influence la perception des problèmes et des risques associés à une mesure et une quantification des phénomènes discriminatoires. Le principe d'égalité, censé être respecté par les institutions publiques spécialisées dans le recueil et le traitement de données statistiques, fait de ces dernières les plus légitimes en matière d'immigration: "Les seuls éléments statistiques que nous utilisons, au niveau régional, mais partout je dirais, ce sont ceux de l'Insee." (Directeur régional Acsé-Paca) .

21 La décentralisation n'a donc pas impulsé, du moins dans le domaine des statistiques sur l'immigration, la construction d'outils de mesure ad hoc concernant les territoires régionaux. Les administrations locales n'ont pas d'autres outils que les statistiques administratives, pour lesquelles seul compte le critère de la nationalité, et cela donne lieu à des insatisfactions : "On ne peut voir que ceux qui sont de nationalité étrangère, et c'est un gros problème car ça fait cinq ans que je travaille sur la lutte contre les discriminations à l'emploi et on ne peut pas évaluer l'impact que ça fait sur le territoire." (Directeur ex-DDTEFP 13).

Il s'agit donc davantage de se réapproprier des données statistiques nationales dans le cadre d'une action publique régionale que de fabriquer territorialement les chiffres à utiliser pour justifier la mise en œuvre de mesures locales.

Le cas des observatoires régionaux ${ }^{16}$ est intéressant à ce sujet car à travers eux s'exprime un besoin de production localisé de statistiques, qui reste pour l'instant très discret dans le domaine de l'immigration. L'entrée légitimante se confirme être celle des discriminations, et non celle de l'immigration en tant que telle: en 2011, la Direction régionale de la jeunesse et des sports et de la cohésion sociale en Paca a lancé l'idée de la création d'un Observatoire régional sur les discriminations, dont l'étude de faisabilité à été confiée à l'ORM (Observatoire régional des métiers), qui est l'observatoire régional de l'emploi et de la formation de la région Paca. Le besoin de capitaliser les expériences passées se combine avec celui de se doter régionalement d'instruments statistiques capables d'appréhender les phénomènes discriminatoires dans leurs multiples facettes et d'outiller les décisions publiques régionales en la matière, en les légitimant.

24 Il s'agit d'un défi toujours actuel en France, car si les statistiques sur les phénomènes migratoires ne sont pas censées résoudre en soi les problèmes des populations qu'elles décrivent, elles sont tout de même en mesure de construire de nouvelles représentations des problèmes ${ }^{17}$. Il sera en ce sens intéressant de voir quels seront les “ entrepreneurs d'action publique ${ }^{18 "}$ qui deviendront protagonistes de cette nouvelle scène 
régionale et s'ils parviendront, comment et avec quels moyens, à remodeler cette action publique en innovant par l'outil statistique.

\section{Le miroir italien : statistiques "mon amour" !} par les acteurs de l'action publique afin de prouver le besoin d'intervenir sur un problème (comme le manque d'hébergements, le travail non déclaré et les accidents du travail) sur lequel l'État ou les collectivités territoriales ne donnent pas une réponse satisfaisante. L'action publique en région Ligurie se distingue donc de façon claire de la région Paca quant à son pouvoir de rendre visibles et légitimes différentes catégories d"'immigrés étrangers".

\section{Conclusion} distinct de l'outil statistique en France et en Italie. En Italie, il représente une entrée parmi d'autres pour intervenir sur la relation emploi-formation, venant justifier le financement de dispositifs proactifs en faveur des populations immigrées.

En France, la discrimination rentre d'abord dans un projet politique surplombant, visant à traiter des problèmes sans nommer les publics, ce qui permet de contourner la mise en place d'actions de discrimination positive ${ }^{21}$ et la construction d'outils de 
mesure spécifiques : l'approche et les instruments adoptés viennent du national pour se décliner territorialement en actions davantage tournées sur la prévention des discriminations.La comparaison internationale nous a permis de contextualiser la construction et l'usage de la catégorie d'“immigré" comme instrument de l'action publique $^{22}$. Elle permet notamment de montrer que les catégories construites au niveau régional ne sont pas toujours le fruit d'opérations cohérentes et délibérées supportées par des modes de coordination performants ${ }^{23}$, et que leur usage est situé car il dépend du contexte sociétal dans lequel elles s'inscrivent. On rejoint en ce sens Gilles Frigoli lorsqu'il souligne comment l' 'émergence de 'nouvelles migrations' agit comme une contrainte exogène qui déstabilise de nombreux acteurs de l'action publique et face à laquelle les pratiques locales constituent une tentative de réponse adaptative ${ }^{24 "}$.

\section{NOTES}

1. Elle a été soutenue le 23 novembre 2011 au laboratoire d'Économie et de sociologie du travail (Aix-en-Provence). Ce travail comparatif a été dirigé par Éric Verdier (LEST, Aix-Marseille Université) et codirigé par Maurizio Ambrosini (université de Milano), et il s'intitule "L"immigré(e) travailleur(se)". La construction et les usages sociaux des catégories de l'action publique en France et en Italie".

2. Nous la concevons comme l'ensemble des activités entreprises par les acteurs sociaux concernés par la conception et la résolution d'un problème public, ce qui nous amène à considérer les formes de coordination des acteurs et les territoires de leur intervention. Voir Pierre Lascoumes, Patrick Le Galès, Sociologie de l'action publique, Paris, Arman Colin, 2007.

3. Il s'agit de la région Provence-Alpes-Côte d'Azur pour la France et de la région Ligurie pour l'Italie. Les deux régions sont limitrophes, tout en étant des espaces privilégiés d'entrée et/ou d'installation d'immigrés (étrangers ou non) pour chacun des deux pays.

4. Didier Fassin (dir.), Les Nouvelles frontières de la société française, Paris, La Découverte, 2010.

5. Nous faisons ici référence aux travaux de Robert Castel, notamment aux Métamorphoses de la question sociale, une chronique du salariat (Paris, Fayard, 1995), et à La Montée des incertitudes (Paris, Seuil, 2009) où l'auteur construit sociologiquement le concept de statut, en en montrant le caractère évolutif.

6. Jean-Pierre Gaudin, Gouverner par contrat, l'action publique en question, Paris, Presses de Science Po, 1999.

7. Alain Morice, Swanie Potot (dir.), De l'ouvrier immigré au travailleur sans papiers. Les étrangers dans la modernisation du salariat, Paris, Karthala, 2010.

8. Alejandro Portes, "La mondialisation par le bas. L'émergence des communautés transnationales", in Actes de la recherche en sciences sociales, $\mathrm{n}^{\circ} 129,1999, \mathrm{pp} .15-25$; Steven Vertovec, "Conceiving and Researching Transnationalism", in Ethnic and Racial Studies, vol. 22, $\mathrm{n}^{\circ}$ 2, 1999, pp. 447-462 ; Maurizio Ambrosini (dir.), Intraprendere tra due mondi : il transnazionalismo economico dei migranti, Bologna, Il Mulino, 2009.

9. Saskia Sassen, "Immigration and local labor markets", in Alejandro Portes (dir.), The Economic Sociology of Immigration. Essays on Networks, Ethnicity, and Entrepreneurship, New York, Russel Sage Foundation, 1995, pp. 87-127.

10. Didier Fassin (dir.), Les Nouvelles frontières de la société française, op. cit. 
11. Alain Desrosières, Laurent Thévenot, Les Catégories socio-professionnelles, Paris, La Découverte, 1988.

12. Emmanuelle Santelli, Grandir en banlieue. Parcours et devenir de jeunes Français d'origine maghrébine, Paris, CIEMI, 2007.

13. Éric Verdier, "Reintroducing public action into societal analysis", in Marc Maurice, Arndt Sorge (dir.), Embedding organizations: societal analysis of actors, organizations and socio-economic context, Amsterdam, John Benjamins publishing company, 2000, pp. 325-338.

14. Bruno Théret, "Méthodologie des comparaisons internationales, approches de l'effet sociétal et de la régulation: fondements pour une lecture structuraliste des sytèmes nationaux de protection sociale", in L'Année de la régulation, vol. 1, 1997, pp. 163-228.

15. Alain Desrosières, Gouverner par les nombres, Paris, Presses de l'École des mines, 2008.

16. Aisling Healy, Éric Verdier, "Dispositifs de connaissance et action publique en région: les Observatoires régionaux de l'emploi et de la formation. Provence-Alpes-Côte d'Azur versus Rhône-Alpes", in Revue française de socio-économie vol. 1, n 5, 2010, pp. 141-164.

17. Alain Desrosières, La Politique des grands nombres. Histoire de la raison statistique, Paris, La Découverte, 2010.

18. Gilles Jeannot, Les Métiers flous : travail et action publique, Toulouse, Octarès, 2005.

19. Richard Hull, Jane Gibbon, Oana Branzei, Helen Haugh, The Third Sector. Dialogues in Critical Management Studies, vol. 1, Emerald Books, 2011.

20. Corrado Bonifazi, Gerardo Gallo, Salvatore Strozza, Donatella Zindato, "Popolazioni straniere e immigrate: definizioni, categorie e caratteristiche", in Studi Emigrazione, n 171, 2008, pp. 519-548.

21. Daniel Sabbagh, Shany Peer, "French color-blindness in perspective: the controversy over 'statistiques ethniques"', in French Politics, Culture, and Society, vol. 26, n 1, 2008, pp. 1-6.

22. Pierre Lascoumes, Patrick Le Galès, Gouverner par les instruments, Paris, Presses de Science Po, 2005.

23. Bénédicte Zimmermann, "Eléments pour une socio-histoire des catégories d' action publique", in Danny Trom, Pascale Laborier (dir.), Historicités de l'action publique, Paris, PUF, 2003, pp. 241-258.

24. Gilles Frigoli, "Les usages locaux des catégories de l'action publique face aux situations migratoires", in Migrations Société, vol. 22, n 128, 2010, p. 90.

\section{AUTEUR}

\section{ANNALISA LENDARO}

Sociologue, post-doctorante à l'École nationale d'administration publique du Canada, Chaire de recherche sur l'évaluation des actions publiques à l'égard des jeunes et des populations vulnérables (Crévaj), Montréal 